\title{
Guided nucleation of superconductivity on a graded magnetic substrate
}

\author{
M. V. Milošević, ${ }^{1}$ W. Gillijns, ${ }^{2}$ A. V. Silhanek, ${ }^{2}$ A. Libál,${ }^{1}$ V. V. Moshchalkov ${ }^{2}$ and F. M. Peeters ${ }^{1}$ \\ ${ }^{1}$ Departement Fysica, Universiteit Antwerpen, Groenenborgerlaan 171, B-2020 Antwerpen, Belgium \\ ${ }^{2}$ INPAC - Institute for Nanoscale Physics and Chemistry, \\ Nanoscale Superconductivity and Magnetism \& Pulsed Fields Group, \\ Katholieke Universiteit Leuven, Celestijnenlaan 200 D, B-3001 Leuven, Belgium
}

(Dated: May 12, 2010)

\begin{abstract}
We demonstrate the controlled spatial nucleation of superconductivity in a thin film deposited on periodic arrays of ferromagnetic dots with gradually increasing diameter. The perpendicular magnetization of the dots induces vortex-antivortex molecules in the sample, with the number of (anti)vortices increasing with magnet size. The resulting gradient of antivortex density between the dots predetermines local nucleation of superconductivity in the sample as a function of the applied external field and temperature. In addition, the compensation between the applied magnetic field and the antivortices results in an unprecedented enhancement of the critical temperature.
\end{abstract}

PACS numbers: 73.23.-b, 74.78.Na

The enhancement of the superconducting critical parameters is arguably the most prominent objective of mesoscopic superconductivity. For example, it is already well established that critical magnetic fields rise in submicron samples to values substantially larger than those found in bulk materials [1], and both critical field and current can be enhanced by nanoengineered periodic [2] and quasi-periodic pinning [3].

The best current method of preventing the deterioration of the superconducting state under external magnetic fields consists of locally counteracting the applied field by an array of ferromagnets with perpendicular anisotropy. These field-compensation effects can lead to a substantial increase of the $T_{c}(H)$ boundary in a field range determined by the magnetization of the dots [4]. More precisely, the maximum of the $T_{c}(H)$ boundary shifts away from zero field, to a value determined by the number of antivortices in the sample [5]. Namely, if magnets are capable of generating vortex-antivortex molecules in the superconductor [6], an applied field that provides exactly the same number of vortices will lead to a complete vortex-antivortex annihilation between the dots, which will maximize $T_{c}(H)$.

In an attempt to achieve the ultimate field compensation, here we study a thin superconducting film evaporated on top of a square array of out-of-plane magnetized

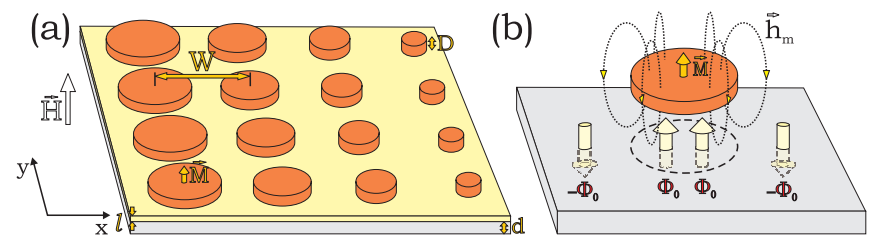

FIG. 1: (color online) (a) A square lattice of magnetic dots with linearly increasing size on top of a superconducting film and an oxide layer. (b) The stray field of each dot $\left(\vec{h}_{m}\right)$ may induce vortex-antivortex molecules in the superconductor [6] . dots of variable size, i.e. parallel periodic rows of dots increasing in diameter [see Fig. 1(a)]. We expect that such gradual magnetic texturing will enhance superconductivity in different parts of the sample at different applied fields, and therefore should lift the $T_{c}(H)$ characteristics as a whole. Since vortex-antivortex $(\mathrm{V}-\mathrm{Av})$ physics is fundamentally important in such a system where different dots induce different numbers of $\mathrm{V}-\mathrm{Av}$ pairs [see Fig. [1(b)], we also investigate the resulting structure of $\mathrm{V}-\mathrm{Av}$ complexes.

Our sample is a $50 \mathrm{~nm}$ thick Al film with critical temperature $T_{c 0}=1.350 \mathrm{~K}$. Experimentally estimated values of coherence length and penetration depth are $\xi(T=0)=117 \mathrm{~nm}$ and $\lambda(0)=98 \mathrm{~nm}$, respectively. A $5 \mathrm{~nm}$ thick Si insulating layer separates the $\mathrm{Al}$ film from the underlying square array of circular magnetic dots with $W=2 \mu \mathrm{m}$ lattice spacing [see Fig. 1(a)], so that any proximity effect can be neglected. The ferromagnetic dots have diameters of $0.2,0.4,0.6,0.8$ and $1.0 \mu \mathrm{m}$ and consist of a $2.5 \mathrm{~nm} \mathrm{Pt}$ buffer layer covered with a [0.4 $\mathrm{nm} \mathrm{Co} / 1.0 \mathrm{~nm} \mathrm{Pt}]_{10}$ multilayer with magnetization perpendicular to the sample surface. Our numerical simulations are performed both within the Ginzburg-Landau (GL) formalism and by molecular dynamics (MD) using vortex-vortex and magnetic dot-vortex interaction potentials derived from London theory [7]. For thin superconducting films (always effectively type-II) the results of these two theories should converge.

Vortex-antivortex complexes To analyze theoretically the vortex-antivortex configuration and their influence on the S/N phase boundary, we employ the GL theory. The two GL equations for the order parameter $\Psi$ and the vector potential A are solved self-consistently, as detailed in Ref. [6]. The ground-state vortex configurations are determined by comparing the Gibbs free energy $\mathcal{F} / \mathcal{F}_{0}=V^{-1} \int\left(2\left(\mathbf{A}-\mathbf{A}_{0}\right) \mathbf{j}-|\Psi|^{4}\right) d \mathbf{r}$ of all found stable vortex states, where $\mathcal{F}_{0}=H_{c}^{2} / 8 \pi$, and all quantities are dimensionless. Here, $\mathbf{j}$ denotes the supercurrent density, 


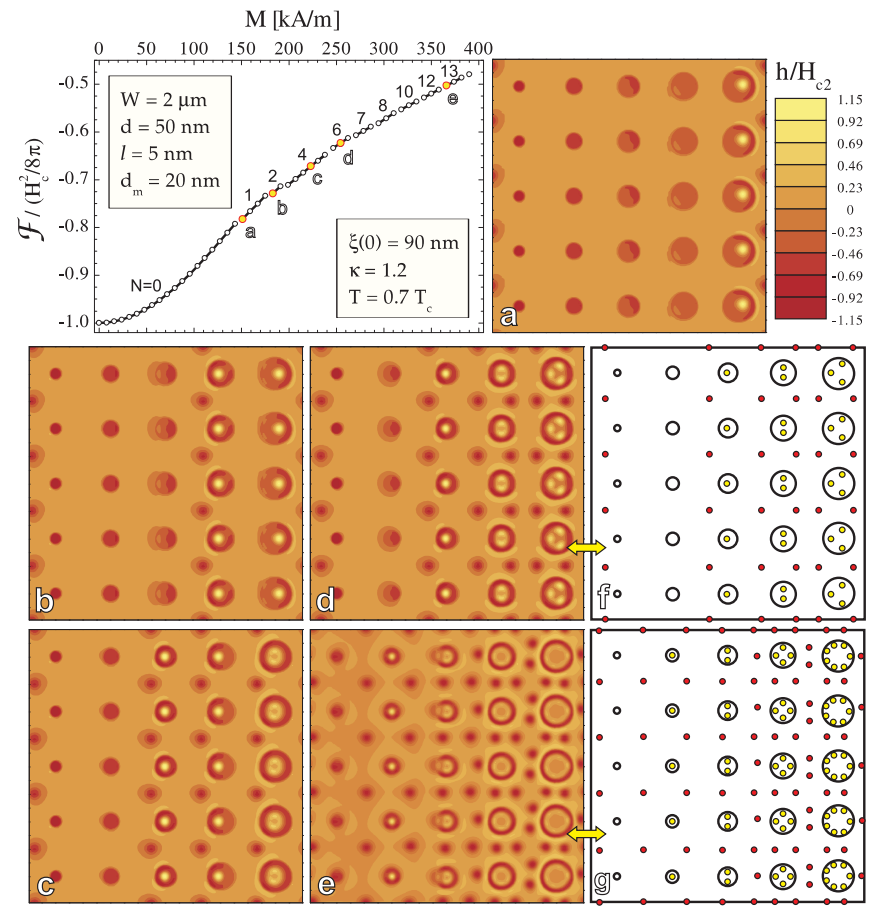

FIG. 2: (color online) The GL free energy of the superconducting state as a function of the magnetization of the magnetic dots (field-cooled regime). (a-e) Local magnetic field distribution in the sample for representative vortex-antivortex configurations $(N=1,2,4,6,13$ antivortices per row respectively), and comparison with MD simulations (f,g).

$A_{0}$ the applied vector potential, and $V$ the sample volume. In what follows, most of the calculated quantities will be expressed (back) in real units for direct comparison with experiment.

Fig. 2 shows the calculated evolution of the groundstate energy of the superconducting film as a function of the magnetization of the magnetic dots, for other parameters taken as in the experiment $\left(d, l\right.$, and $d_{m}$ denote the thickness of the film, oxide and the magnetic dots respectively). As schematically shown in Fig. 1(b), each dot generates an inhomogeneous stray field which favors the nucleation of vortex-antivortex $(\mathrm{V}-\mathrm{Av})$ pairs. For a given magnetization, $\mathrm{V}-\mathrm{Av}$ pairs first nucleate under the largest dots, due to their larger magnetic moment [see Fig. 1(a)]. In interaction with other dots, antivortices occupy interstitial positions, while vortices remain under the largest dots at a somewhat off-center position. With increasing magnetization, more $\mathrm{V}-\mathrm{Av}$ pairs are induced in the sample, and they gradually appear around smaller dots as well [see Figs. 2(b-d)]. Increased density of antivortices between the magnetic dots leads to their 'crystallization' into an interstitial lattice [Fig. 2(e)]. Results of the GL theory are nicely corroborated by MD simulations [see Figs. $2(\mathrm{f}, \mathrm{g})]$.

Two important conclusions follow from the above results. First, our gradiated magnetic texture leads to a gradient of antivortex density along the sample (for vortices as well, but they are localized under the magnetic dots). Second, depending on the spacing between the magnetic dots and the number of nucleated antivortices, some of them can penetrate even the regions free of nucleated vortex-antivortex pairs [see area around smallest dots in Fig. 2(e)]. This shows that antivortices can detach from the original magnetic dot and move towards other dots, guided by an overall magnetic potential.

The latter conclusions are even more evident if realistic structural defects are introduced in the calculation. In Fig. 3(a) we show the vortex-antivortex state obtained for $M=300 \mathrm{kA} / \mathrm{m}$, where we allowed for minor deviations of the size of the magnets $(<1 \mathrm{~nm})$ compared to the experimentally suggested values. As a result, some dots with the same nominal size generate different number of $\mathrm{V}-\mathrm{Av}$ pairs in the sample and consequently the overall magnetic profile becomes less uniform and antivortices penetrate easier the areas around the dots of insufficient moment for the nucleation of $\mathrm{V}-\mathrm{Av}$ pairs. Interestingly, the magnetic profile in the sample changes considerably with increasing temperature [see Figs. [3(b-d)]. Fig. 3 shows that higher temperature leads to: (i) appearance of new V-Av pairs around some dots; (ii) merging of confined vortices under the dots into a giant-vortex [8]; (iii) increased rigidity of the antivortex lattice between the magnetic dots; and (iv) gradual destruction of superconductivity, starting from areas around the largest dots. Molecular dynamics simulations disregard the suppression of superconductivity under the magnetic dots and do not take into account the large vortex cores - as a consequence they fail to reproduce the GL results at temperatures above $0.8 T_{c}$.

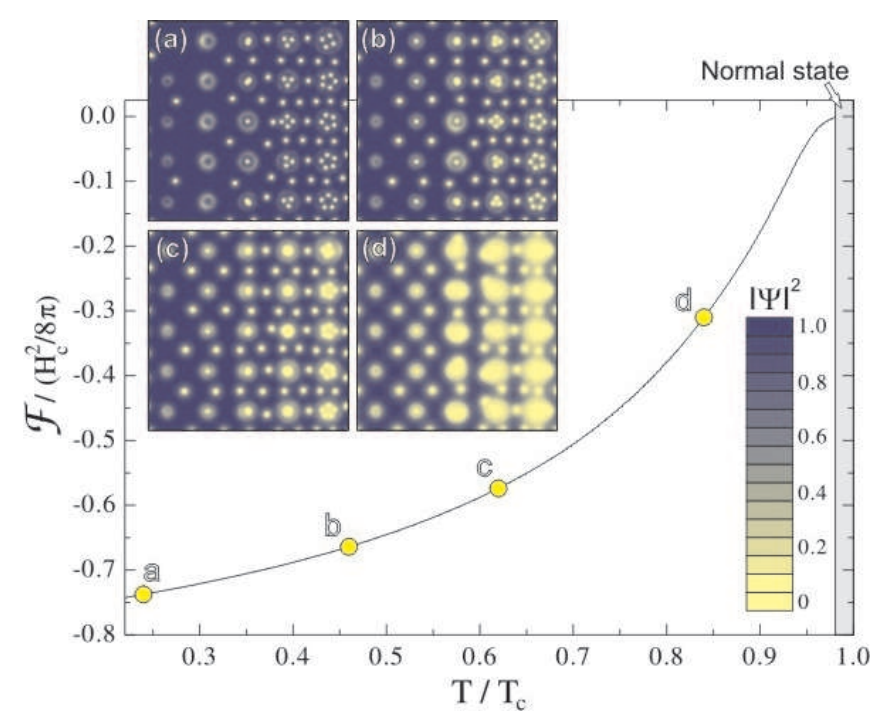

FIG. 3: (color online) The evolution of the Cooper-pair condensate (and its energy) under the gradiated array of magnetic dots as a function of temperature (for $M=300 \mathrm{kA} / \mathrm{m}$ ). 
Controlled nucleation of superconductivity The above findings indicate that with increasing temperature superconductivity is longest preserved in the region around the smallest magnetic dots. However, it is already a well-known fact that nanostructuring of superconducting films by perpendicularly magnetized dots leads to a shift of the $H-T$ phase boundary, a phenomenon known as field-induced-superconductivity [4]. This phenomenon is asymmetric with applied external field, i.e. fields parallel to the moment of magnetic dots enhance superconductivity (due to stray field compensation and vortexantivortex annihilation between the dots) while applied antiparallel field only suppresses superconductivity further. As a result, the $H-T$ boundary shifts with respect to $H=0$, and exhibits maximal $T_{c}(H)$ at a field determined by the magnetic moment of the dots. In our present sample however different rows of magnetic dots have different magnetic moments, and it is by no means obvious how the $H-T$ boundary will look like.

The results of our transport measurements and GL simulations for the superconducting/normal phase $H-T$ boundary are shown in Fig. 4. In the theoretical simulations, the nucleation of superconductivity was monitored locally, i.e. maximal $|\Psi|^{2}>10^{-12}$ was declared as a superconducting state. In the case of negative applied field, we obtained an identical phase boundary for both magnetized and demagnetized dots (dashed curve in Fig. 44). In this case, since superconductivity always nucleates along the line between the rows of the two smallest dots, $T_{c}(H)$ shows an approximately linear behavior. Deeper inside the superconducting phase (lower $T$ ) the applied magnetic field induces additional antivortices in the sample, increasing the configurational complexity. As an illustration, the inset of Fig. [4 at $T=0.86 T_{c}$ and $H=H_{-4}$, i.e. matching field adding 4 antivortices per magnetic dot, shows the resulting structure of both antivortex molecules around the magnetic dots and an antivortex lattice between them.

On the other hand, for applied positive magnetic field we observe a clear enhancement of superconductivity in our sample. As detailed in Ref. [5], the highest $T_{c}$ in magnetically structured superconducting films is always obtained at the applied field matching the number of antivortices per periodic unit cell of the sample. Since our sample contains magnetic dots of five different sizes and correspondingly different number of induced antivortices around them $(N=1+2+2+5+8)$, we achieve the latter condition for maximal $T_{c}$ at several values of applied magnetic field! As a consequence the overall maximum of $T_{c}$ remains at $H=0$, but the $T_{c}(H)$ boundary is lifted tremendously as compared to the reference sample. The superposition of compensation phenomena in different parts of the sample leads to several cusps in $T_{c}(H)$ 9], but the general trend is clear - as the applied magnetic field is increased, superconductivity nucleates first in the regions of the sample with matching antivor-

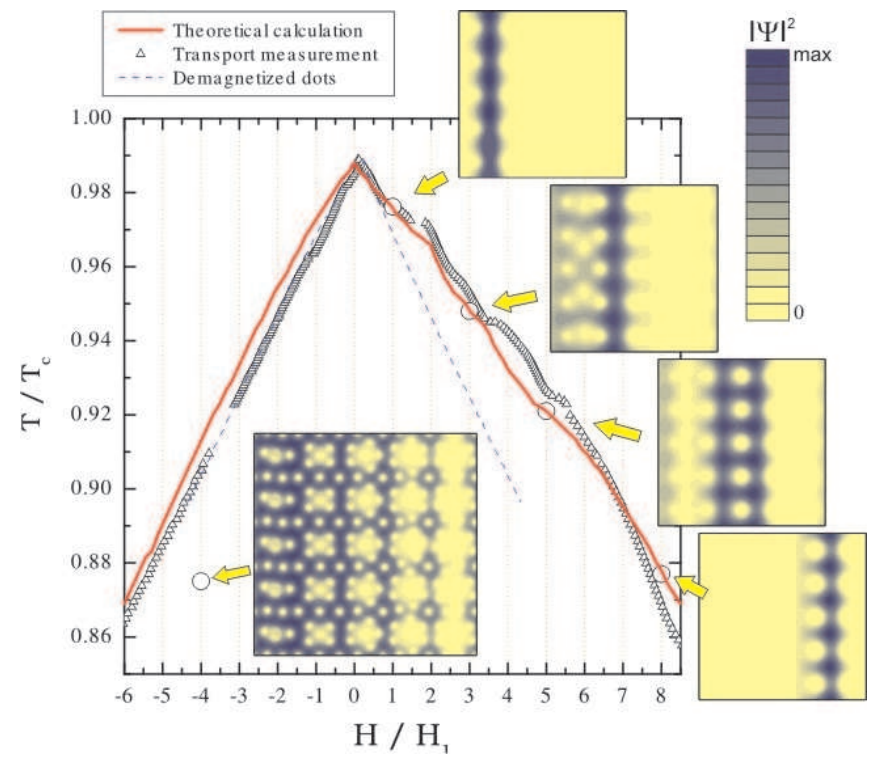

FIG. 4: (color online) The superconducting/normal (S/N) $H-T$ phase boundary obtained by transport measurements, compared to the case of demagnetized dots and the theoretical data (for $M=450 \mathrm{kA} / \mathrm{m}, \lambda(0)=100 \mathrm{~nm}, \xi(0)=110 \mathrm{~nm}$ ). The insets show the Cooper-pair density plots at $(\mathrm{H}, \mathrm{T})$ values denoted by open dots.

tex density. As illustrated in Fig. 4, the nucleation line shifts from smallest (at $H=H_{1}$ ) towards largest dots (for $H=H_{8}$ ). Such guided nucleation of superconductivity is not only a unique fundamental phenomenon, but it is of value for potential multi-channel applications, and, as shown in Fig. 4, leads to an unprecedented enhancement of superconductivity in an applied magnetic field.

To summarize, thanks to the specific design of the arrays of magnetic dots with varied size, and the resulting gradient in antivortex density in the superconducting film, we achieved the highest overall critical temperature of superconducting films in the presence of applied magnetic field. We also show that nanoengineered arrays of magnetic dots enable the control of local nucleation of superconductivity by simply changing the magnitude of applied magnetic field.

This work was supported by the Flemish Science Foundation (FWO-Vl), the Belgian Science Policy (IAP), and the ESF-NES program. W.G., A.V.S. and A.L. acknowledge individual support from FWO-Vlaanderen.

[1] V.V. Moshchalkov, L. Gielen, C. Strunk, R. Jonckheere, X. Qiu, C. Van Haesendonck, and Y. Bruynseraede, Nature 373, 319 (1995).

[2] Q. Niu and F. Nori, Phys. Rev. B 39, 2134 (1989); M. Baert, V.V. Metlushko, R. Jonckheere, V.V. Moshchalkov and Y. Bruynseraede, Phys. Rev. Lett. 74, 3269 (1995); G.R. Berdiyorov, M.V. Milošević, and F.M. Peeters , 
Phys. Rev. B 74, 174512 (2006).

[3] V. Misko, S. Savel'ev, and F. Nori, Phys. Rev. Lett. 95, 177007 (2005).

[4] M. Lange, M.J. Van Bael, Y. Bruynseraede, and V.V. Moshchalkov, Phys. Rev. Lett. 90, 197006 (2003); M.V. Milošević and F.M. Peeters, Europhys. Lett. 70, 670 (2005); A.Yu. Aladyshkin, A.V. Silhanek, W. Gillijns and V.V. Moshchalkov, Supercond. Sci. Technol. 22, 053001 (2009).

[5] W. Gillijns, M.V. Milošević, A.V. Silhanek, V.V.
Moshchalkov, and F.M. Peeters , Phys. Rev. B 76, 184516 (2007).

[6] M.V. Milošević and F.M. Peeters, Phys. Rev. B 68, 024509 (2003); idem. Phys. Rev. Lett. 93, 267006 (2004).

[7] M.V. Milošević and F.M. Peeters, Phys. Rev. B 68, 094510 (2003).

[8] D.S. Golubović, M.V. Milošević, F.M. Peeters, and V.V. Moshchalkov, Phys. Rev. B 71, 180502 (2005).

[9] Resembling commensurability effects, see Ref. [2]. 